\title{
SENTIDOS SOBRE CAMBIO CLIMÁTICO Y SUS RELACIONES CON LA CUESTIÓN AMBIENTAL PRODUCIDOS ENTRE DOCENTES DE CIENCIAS E INVESTIGADORES EN ECOLOGÍA
}

\author{
Raquel dos Santos Moniz Benac ${ }^{1}$ \\ Mariana Brück Gonçalves ${ }^{2}$ \\ Gina Alejandra Huérfano Aguilar ${ }^{3}$ \\ Laísa Maria Freire ${ }^{4}$
}

\section{Resumen}

El presente trabajo, por medio del referencial teórico y metodológico del dialogismo del discurso de Bakthin, busca caracterizar sentidos de Cambio Climático y sus relaciones con la cuestión ambiental, producidos en una interacción. El estudio se realizó durante el desarrollo de un taller de formación continuada, donde los actores participes de la investigación fueron una profesora de ciencias y dos investigadores en ecología. Durante el curso, la dinámica seleccionada para este trabajo fue grabada, transcrita y los datos fueron analizados, constituyendo el corpus de este estudio con dieciocho enunciados. A partir del análisis de los sentidos de los participes, encontramos que el debate entre docentes e investigadores contribuye a una mirada a la cuestión ambiental que profundiza la formación en ciencias, quizás contribuyendo a la generación de un diálogo de saberes y a la inclusión de lo ambiental en las clases de ciencias.

Palabras clave: Educación Ambiental. Cambio Climático. Formación continuada de docentes en ciencias.

\section{MEANINGS OF CLIMATE CHANGE AND ITS RELATIONS TO THE ENVIRONMENTAL ISSUE PRODUCED BETWEEN SCIENCE TEACHERS AND RESEARCHERS IN ECOLOGY}

\begin{abstract}
The present work, through the theoretical and methodological referential of the discursive dialogism of Bakhtin, characterize the meanings of Climate Change and its relations with the environmental question produced in an interaction. The study was conducted during the development of a dynamic of a continuous training course, in which the actors involved in the research were a science teacher and two researchers in ecology. During the course, a dynamic was developed on the environmental issue and its relation with Climate Change. The dynamics selected for this work were recorded, transcribed and the data were analyzed, constituting the corpus of this study with eighteen statements. From the analysis of the senses of the participants, we understand that the interaction between teachers in sciences and researchers in ecology has

\footnotetext{
${ }^{1}$ Mestre, Laboratório de Limnologia, Instituto de Biologia, Programa de Pós-graduação em Ciências Ambientais e Conservação - NUPEM/UFRJ.

${ }^{2}$ Mestre, Laboratório de Limnologia, Instituto de Biologia, Programa de Pós-graduação em Educação em Ciências e Saúde - NUTES/UFRJ.

${ }^{3}$ Mestranda, Laboratório de Limnologia, Instituto de Biologia, Programa de Pós-graduação em Ciências Ambientais e Conservação - NUPEM/UFRJ.

${ }^{4}$ Doutora e Professora, Laboratório de Limnologia, Departamento de Ecologia, Instituto de Biologia Universidade Federal do Rio de Janeiro. Programa de Pós-graduação em Educação em Ciências e Saúde NUTES/UFRJ e Programa de Pós-graduação em Ciências Ambientais e Conservação - NUPEM/UFRJ.
} 
contributed to analyze the environmental issue that deepens the formation in sciences, and can contribute to the generation of a knowledge dialogue and the inclusion of the environmental issue in science classes.

Keywords: Environmental education. Climate Change. Continuing education of teachers in science.

\section{SENTIDOS SOBRE MUDANÇAS CLIMÁTICAS E SUAS RELAÇÕES COM A QUESTÃO AMBIENTAL PRODUZIDOS ENTRE DOCENTES EM CIÊNCIAS E PESQUISADORES EM ECOLOGIA}

\section{Resumo}

O presente trabalho, através do referencial teórico e metodológico do dialogismo discursivo de Bakhtin, busca caracterizar os sentidos das Mudanças Climáticas e suas relações com a questão ambiental produzidos em uma interação. $\mathrm{O}$ estudo foi realizado durante o desenvolvimento de uma dinâmica de um curso de formação continuada, em que os atores envolvidos na pesquisa foram um professor de ciências e dois pesquisadores em ecologia. Durante o curso, desenvolveu-se uma dinâmica sobre a questão ambiental e sua relação com Mudanças Climáticas. A dinâmica selecionada para este trabalho foi registrada, transcrita e os dados foram analisados, constituindo o corpus deste estudo com dezoito enunciados. A partir da análise dos sentidos dos participantes, entendemos que a interação entre professores de ciências e pesquisadores em ecologia contribuiu para uma análise da questão ambiental que torna mais profunda a formação em ciências, podendo contribuir com a geração de um diálogo de saberes e a inclusão do tema ambiental nas aulas de ciências.

Palavras-chave: Educação Ambiental. Mudança Climática. Formação continuada de docentes em ciências.

\section{Introducción}

El surgimiento de la cuestión ambiental ${ }^{5}$ como parte del currículo de la enseñanza de las ciencias ha sido resultado de las reflexiones contemporáneas sobre la manera en que la sociedad comprende y se relaciona con la naturaleza. A partir de los años 1970, la cuestión ambiental se pone de relieve en la sociedad y, posteriormente, llega los procesos educativos.

En este contexto surge la Educación Ambiental (EA), proveniente de los movimientos ambientalistas, que busca, en sus primeros intentos, una actuación frente a los problemas ambientales y una cierta reintegración entre el ser humano, la sociedad con la naturaleza. La EA, por tratarse de un proceso pedagógico (con distintas concepciones), que reflexiona sobre la cuestión ambiental, la podemos concebir como un medio en que el tema ambiental es introducido en el contex to escolar (LAYRARGUES; LIMA, 2014).

Por otra parte, cada vez más se discute el rol de la ciencia y su relación con la cuestión ambiental en la contemporaneidad, porque al mismo tiempo en que la ciencia se coloca como un medio de 'solucionar' los problemas ambientales de la actualidad, se cuestiona su función en

5 La cuestión ambiental es entendida, en este capítulo, a partir de sus múltiples dimensiones: sociales, políticas, económicas, naturales y culturales, siendo su surgimiento a partir de las reflexiones sobre la relaciones entre el ser humano y la naturaleza - demarcado históricamente en los años 70, pelo aparecimiento de la percepción de que lo crecimiento económico ilimitado (lo cual explora y domina lo medioambiente) no era sustentable. Por lo tanto, la cuestión ambiental va más allá de solo problemas relacionados a los sistemas naturales, ya que en esta se involucra cuestionamientos sobre las desigualdades e injusticas sociales y las transformaciones territoriales y culturales producidas por lo sistema económico vigente. 
el modelo económico actual, ya que a la vez puede servir para mantener y exacerbar las desigualdades sociales, así como la distribución de los impactos y riesgos ambientales entre los distintos grupos sociales (VENTURA; FREIRE, 2015). Por tanto, consideramos que la cuestión ambiental debe ser puesta de relieve en la enseñanza de las ciencias y que cada vez más la necesidad de comprensión de la cuestión ambiental, debe generar cambios en procesos de formación de docentes en ciencias.

El objetivo del presente trabajo fue caracterizar sentidos sobre Cambio Climático (CC) y sus relaciones con la cuestión ambiental, producidos en el diálogo entre una profesora de ciencias e investigadores en ecología. Para eso, en un primer momento, discutimos aspectos de la cuestión ambiental en la contemporaneidad y del CC, caracterizando su abordaje en la EA e Educación en Ciencias (EC). En un segundo momento, abordamos el referente teóricometodológico sobre estudios del lenguaje, a través de los aportes del dialogismo de Bakthin. En un tercer momento, se desarrolla la metodología abordada en el estudio, dando a conocer el contexto, el diseño del taller de formación, los actores partícipes y los métodos de recolecta de datos, y finalmente, hacemos análisis de los datos recogidos. A modo de síntesis, discutimos las relaciones entre sentidos del CC durante la interacción, lo que nos permitió reflexionar sobre cómo la EA y la EC pueden relacionarse en contextos particulares (del taller realizado) y cómo la EA puede contribuir a la discusión de las cuestiones ambientales en la EC.

\subsection{La cuestión ambiental en la contemporaneidad y el cambio climático}

La relación entre el ser humano y la naturaleza ha generado impactos ambientales, que se distribuyen entre los distintos actores sociales y territorios de forma desigual: la degradación de los ecosistemas, la sequía, la escasez y la falta de acceso al agua, las epidemias, la contaminación, la pérdida de la biodiversidad y el patrimonio cultural, el aumento de la temperatura, etcétera. (FREIRE; CÁCERES; JULIANI, 2016). En este contexto, las soluciones de la ciencia y de la tecnología vinculadas al desarrollo económico se consideran, cada vez más, como posibilidades para enfrentar una crisis ambiental.

Sin embargo, algunas preguntas surgen cuando consideramos la ciencia como una producción humana y las contradicciones del sistema económico en vigor: ¿Cómo la ciencia con una concepción fragmentada del mundo puede abordar la complejidad de la cuestión ambiental? Cómo el capitalismo puede abordar los problemas que ha creado y crea - la degradación ambiental, la explotación de los territorios, las desigualdades y las injusticias? (VENTURA; FREIRE, 2015; LEFF, 2009).

Con esto, la relación entre el medio ambiente y la ciencia se hace más evidente, así como la necesidad de reflexionar sobre cómo entendemos esta relación y, también, de investigar sobre las formas que se colocan como posibles soluciones a la crisis ambiental. Así, estas demandas contemporáneas para un enfrentamiento justo de la cuestión ambiental conllevan a la inclusión del tema ambiental en el currículo de ciencias.

El CC se configura como una cuestión ambiental contemporánea, compleja por presentar múltiples intereses en conflicto, en el campo científico (escépticos versus defensores), en las esferas política, social, económica, y cultural (foros de discusión en favor de resoluciones del CC que legitiman los discursos y el conocimiento de determinados grupos, invalidando el de los otros; las disputas entre países del Norte y del Sur; las desigualdades en la distribución de los riesgos y la vulnerabilidad socioambiental entre los distintos territorios del mundo; el uso de la tierra por intereses económicos) (CASAGRANDE; SILVA JR.; MENDONÇA, 2001; LIMA, 2009; LIMA, 2013). Es necesario saber que no se conoce una solución sencilla para el enfrentamiento de posibles impactos generados por el CC en la sociedad actual, dado que la ciencia no es la única a aportar soluciones para su enfrentamiento. 
Asimismo, las posibles soluciones sugeridas por la ciencia (vinculadas a reducción y secuestro de los gases que causan el CC, así como soluciones de mitigaciones) y legitimadas como las más consistentes por áreas de política (foros, COPS, planes de mitigación etc.), mediáticas (alarmismo, ocultamiento de lo debate de las controversias del CC, así como de las visones entre Norte y Sul global sobre este problema, y anuncio de soluciones solo económicas) y económicas (crédito de carbono, energías renovables, cambio en padrones individuales de consumo y mercado verde) como un medio para hacer frente a lo CC, no pueden dar cuenta de la resolución de un complejo y polémico tema. Esto porque en el proceso de mitigación de los impactos del CC hay elementos ocultos y desconocidos por la propia ciencia, además de una diversidad de aspectos que envuelven su enfrentamiento, relacionados a las distintas percepciones de los distintos actores sociales involucrados, los intereses políticos y económicos ocultos en distintos discursos que rodean este asunto (LIMA, 2009).

\subsection{La Educación Ambiental y Educación en Ciencias en la formación de docentes en ciencias para el abordaje de cuestiones ambientales contemporáneas}

Segundo Guimarães e Vasconcellos (2006), el vínculo entre EA y EC en procesos formativos y en la práctica docente de los profesores de ciencias (correlacionando la complementariedad entre las diferentes esferas de la educación, tanto formal como informal) puede revelar los diferentes discursos que circulan sobre la cuestión ambiental y la ciencia, así como aumentar la participación política de las diferentes partes interesadas para una transformación social, además de incluir las preguntas: ¿Cómo se construye el conocimiento? ¿Cuáles son los motivos de legitimar algunos saberes mientras otros son subyugados?. En cuanto para Tozoni-Reis (2002) los propios significados atribuidos a la relación sociedadnaturaleza están vinculados en la interfaz entre EA y EC, cuando revela que hay discursos que empoderan los sujetos y la colectividad, que están relacionados con una perspectiva de transición de paradigmas de conocimiento en un intento de superar el paradigma científico.

Igualmente, entendemos que los diversos sentidos acerca de las interrelaciones de los diversos aspectos de la cuestión ambiental, se plantean en relación a cómo la EA ha sido significada a lo largo de la historia (y establece relaciones con la ciencia, en la forma en que entendemos y producimos el conocimiento). Por lo tanto, podemos entender la importancia de la vinculación entre EA y EC en procesos de formación de profesores de ciencias por los desafíos de los complejos problemas ambientales contemporáneos (TOZONI-REIS, 2008).

En ese contexto, la EA en la formación de docentes de ciencias puede contribuir a la comprensión (y en la revelación) sobre la relación sociedad-naturaleza y potencializar la creación de espacios dialógicos para el enfrentamiento de cuestiones ambientales contemporáneas. Desde esta perspectiva, la EA aliada a la EC puede ayudar con el tratamiento didáctico del $\mathrm{CC}$, en el ámbito pedagógico, mediante una formación que cuestiona la relación entre la sociedad y la naturaleza, los paradigmas del conocimiento y, al mismo tiempo, empodera para luchas políticas y socioambientales relacionadas a este tema, así como sus propios problemas éticos (tales como la distribución de los impactos socioambientales desiguales).

En la esfera de la acción comunitaria, la EA podría actuar a través de la capacitación para la participación y para el diálogo entre distintos actores sociales, en las distintas dimensiones que involucran el CC (LIMA, 2013; SAUVE, 2010). Además, se puede descubrir cómo los diferentes actores sociales involucrados significan el tema, revelando la relación entre la epistemología y política, entre saber y poder, mostrando que hay conocimientos instrumentales al servicio de la dominación social y el medio ambiente, a su vez mostrando que existen saberes comprometidos con la emancipación de estas relaciones. (LIMA, 2013). 
Así, el vínculo entre EA y EC en la formación de profesores de ciencias puede contribuir para que estos actúen en los procesos educativos, promoviendo una visión crítica del conocimiento científico - revelando su construcción histórica y social - así como, los distintos discursos pronunciados por los investigadores influyen en la producción del conocimiento científico, en cuestiones éticas (relacionados con las injusticias ambientales), así como capacitando a sus estudiantes con argumentos para tratar estas diferentes visiones y el empoderamiento político sobre la base de la ciudadanía y la justicia ambiental para lo enfrentamiento de temas ambientales contemporáneos (SAUVE, 2010; FREIRE; CÁCERES, JULIANI, 2016).

\section{Dialogismo del discurso de Bakthin}

En el presente trabajo utilizamos, como marco teórico, el dialogismo del discurso de Bakthin. Para este autor, es en la interacción social que el lenguaje es constituido, así como es el sujeto, los discursos y los sentidos. Más específicamente, trabajamos con el concepto de enunciado para entender los sentidos de CC en una interacción social.

Para Voloshinov yBakhtin (1976), el enunciado consiste en una actividad del lenguaje $\mathrm{y}$, simultáneamente, una actividad humana, en el que el leguaje es intrínseco a la sociedad. Relacionando así, en su teoría, el lenguaje con las prácticas sociales. El en cuando concreto, se constituye de lo percibido y del presumido (los actos sociales compartidos por un determinado grupo). Siendo el contexto extraverbal los conjuntos comunes de lo conocido, el entendido y del evaluado por los participantes, y que vivifica lo verbal en algo con significado pleno para el otro.

La noción del dialogismo, en Bakhtin, se refiere a los propios conflictos y luchas de voces dentro del enunciado, donde el yo sufre influencia directa del otro y la significación del enunciado solo es posible en la interacción entre lo que habla y lo que recibe. (VOLOSHINOV; BAKHTIN, 1976; BAKHTIN, 2006).

Además, aquel que enuncia siempre buscará evaluar a quien se destina sus enunciados, de acuerdo con el contexto de la interacción, las cuestiones relacionadas a las posiciones jerárquicas y sus convicciones. Siendo la cultura constituida por los discursos que retienen la memoria colectiva y en relación a cual es necesaria una toma de posición. Es esa interacción dialógica y de opiniones que genera el movimiento y las transformaciones, alejando el sujeto de la subordinación (PIRES, 2002).

La noción de diálogo se basa en la característica activa y de respuesta al enunciado, dado que el hablante busca respuestas que enseñen adhesión, concordancia o contrariedad a las ideas expuestas, y a la vez implica un juicio de valor que parte de la relación del enunciado con la realidad, con el hablante y con otros enunciados anteriores. Estas relaciones conforman los elementos ideológicos en los discursos (PIRES, 2002; BAKHTIN, 2006).

A partir de los enunciados, se llega a la significación de los resultados. El esfuerzo de comprensión de los enunciados permite la significación de los mismos. El sentido del enunciado es, también, engendrado por las condiciones reales de la enunciación y se distribuye entre las diversas voces que habitan el tejido del lenguaje. Se establece, así, un relacionamiento dialógico de sentidos entre enunciados confrontados. Las relaciones dialógicas son relaciones semánticas entre los enunciados en la comunicación verbal (SOUZA; ALBUQUERQUE, 2012). Los sentidos atribuidos por los autores, por determinado tema, se construyen en la interacción social y para la comprensión del enunciado las condiciones de los que hablan deben ser consideradas (SÁ et al., 2014). 


\section{El contexto de la investigación}

El contexto de esta investigación consistió en una dinámica desarrollada durante un taller de formación para profesores de ciencias con el tema del CC en la perspectiva de la EA. Dicho taller se configura como una propuesta de interacción entre los investigadores en ecología y profesores de ciencias con la mediación de investigadores en educación en ciencias y la EA. Además, el taller es parte de un proyecto de investigación que fue aprobado por el Comité de Ética de Investigación del Instituto de Estudios de Salud Colectiva de la UFRJ y parte del proyecto de extensión de la universidad: Universidade-Escola - Cursos de formación, capacitación y actualización a los maestros de los municipios y el estado de Macaé y región (una colaboración entre el sector de extensión social de UFRJ (NUPEM/UFRJ en Macaé, Rio de Janeiro, Brasil) con la Consejería de Educación de la municipalidad de Macaé SEMED/CPROFOR.

El taller se dividió en cinco sesiones de cuatro horas, en el parque municipal de medio ambiente de Macaé. La descripción de cómo hemos trabajado los temas en cada sesión está en el Cuadro 1.

Cuadro 1 - Tema duración y descripción de las actividades de las sesiones del taller

\begin{tabular}{|c|c|c|c|}
\hline Sesión & Tema & Descripción de los temas de cada sesión & Horas \\
\hline (1) & Introducción al $C C$ & $\begin{array}{l}\text { En esta sesión fue presentada la propuesta de } \\
\text { diálogo de saberes en el tema CC entre los } \\
\text { profesores en ciencias y los investigadores en } \\
\text { ecología, por otro lado, se desarrollaron } \\
\text { dinámicas para la aproximación entre los } \\
\text { participantes y una presentación introductoria } \\
\text { sobre el CC por un investigador. }\end{array}$ & $4 \mathrm{~h}$ \\
\hline (2) & $\begin{array}{l}\text { Controversias } \\
\text { sociocientíficas en el } \\
\text { tema sobre el CC y } \\
\text { sus relaciones con } \\
\text { EA y EC }\end{array}$ & $\begin{array}{l}\text { En esta sesión se abordó las controversias del } \\
\text { CC por medio de una dinámica de grupos y } \\
\text { con una presentación magistral. Además, } \\
\text { hemos hecho un sendero con los participantes } \\
\text { y una actividad de cartografía social. }\end{array}$ & $4 \mathrm{~h}$ \\
\hline (3) & $\begin{array}{l}C C \text { en Ecosistemas } \\
\text { del Norte Fluminense }\end{array}$ & $\begin{array}{l}\text { En esta sesión se buscó contextualizar la } \\
\text { cuestión ambiental en lo contexto del CC por } \\
\text { medio de una actividad entre los profesores e } \\
\text { investigadores, seguida de una presentación } \\
\text { de las investigaciones en ecología } \\
\text { relacionadas a este tema y, finalmente, una } \\
\text { actividad de producción de textos con los } \\
\text { profesores. }\end{array}$ & $4 \mathrm{~h}$ \\
\hline (4) & $\begin{array}{lllll}C C & y & E A & \text { en } & \text { el } \\
\text { contexto escolar }\end{array}$ & $\begin{array}{l}\text { En esta sesión fue desarrollada una actividad } \\
\text { sobre el CC en el contexto escolar, así como } \\
\text { también se presentó a los participantes como } \\
\text { se elabora una secuencia didáctica, ya que } \\
\text { nuestra intención fue estimular el diálogo de } \\
\text { saberes entre los profesores y los } \\
\text { investigadores para la elaboración conjunta de } \\
\text { tal secuencia sobre el CC y sus relaciones } \\
\text { entre EA y EC }\end{array}$ & $4 \mathrm{~h}$ \\
\hline (5) & $\begin{array}{l}\text { Elaboración } \\
\text { conjunta de } \\
\text { secuencia didáctica }\end{array}$ & $\begin{array}{l}\text { La última sesión consistió en el planeamiento } \\
\text { de la secuencia didáctica sobre el CC entre los } \\
\text { profesores e investigadores que fue elaborada } \\
\text { en grupos, así como en la presentación de } \\
\text { estas. }\end{array}$ & $4 \mathrm{~h}$ \\
\hline
\end{tabular}




\subsection{Los actores partícipes de la investigación}

Los actores partícipes de la interacción del taller fueron ocho profesores (tres profesores de ciencias, cuatro futuros docentes de ciencias, un profesor de educación física) y un oyente. Además de participantes, tres investigadores en ecología que trabajan con la temática del CC participaran del taller como docentes del taller y en la investigación como sujetos de la investigación.

A pesar de ocho participantes y tres investigadores, para el análisis detallado de los sentidos de CC, consideramos específicamente para este trabajo los enunciados de dos investigadores en ecología y de una profesora de ciencias. Han sido elegidos solamente aquellos participantes del curso con participación continua en la interacción. Identificamos los actores con nombres fictícios. Los investigadores en ecología fueron llamados de: João y $A n a^{6}$, por otro lado, a la profesora de ciencias llamamos $\operatorname{Marí}^{7}$. Los participantes que actuaron durante la interacción, siendo sus enunciados no considerados en el análisis, pero considerados en los contextos extraverbal y considerados para entender los enunciados analizados: José (investigador), Amanda (futura docente de ciencias), Paulo (docente de educación física) y Pablo (oyente). Los otros participantes en el contexto de la interacción no emitieron enunciados, no obstante, en pocos momentos ellos expresaron algunos gestos o sonrisas en el grupo.

\subsection{La producción de datos y la identificación de los enunciados}

La recogida de datos ocurrió durante una dinámica ocurrida en la tercera sesión del taller, en la cual se discutió el tema ambiental y su relación con el CC. La actividad se desarrolló de la siguiente manera: en primer lugar, la mediadora explicó la dinámica, en la que se invitó a los participantes a reflexionar sobre la cuestión ambiental y sus relaciones con el CC. Para esto, entregamos en cada grupo un texto distinto que evidenciaba la desigualdad de los impactos del $\mathrm{CC}$ a nivel global a través de relatos de personas que sufrían los efectos del CC en distintos lugares del mundo, y un conjunto de tres mapas (todos los grupos recibieron los mismos mapas) que mostraban las desigualdades a través de la distribución de estos impactos entre los distintos territorios.

El texto 1, Comunidades do Cariri cearense, fue adaptado de Lindoso (2013) y presenta una historia de consecuencias del $\mathrm{CC}$ en una región semiárida del Brasil; el texto 2, Comunidades de la geleira Chacaltaya, fue adaptado de Olfarnes y Gitow (2009) y presenta una historia de los impactos del CC que se pasa en Bolivia, en las montañas Huayna Potosí y Chacaltaya. El último texto presenta la historia de Thombi Masondo, que se pasa en Africa del Sur. Las gráficas utilizadas presentaban informaciones de UNESCO (2014) sobre vulnerabilidad costera e impactos relacionados al clima, además de informaciones sobre distribución de empleos, en distintas partes del mundo, por género (femenino y masculino) y por sectores (agricultura, industria y servicios). Todas estas informaciones disponibles podrían permitir a los participantes la construcción de diferentes enunciados del CC, vinculados a áreas políticas, económicas, culturales, etnográficas, científicas etc.

Los participantes fueron divididos en tres grupos (un investigador en ecología en cada grupo) con el propósito de hacer reflexiones sobre las cuestiones planteadas: ¿Qué es la

\footnotetext{
${ }^{6}$ También son los autores-criadores (1), siendo sus destinatarios la mediadora, la profesora de ciencias y los otros participantes.

${ }^{7}$ También é el autor-criador (2), siendo sus destinatarios los investigadores en ecología, la mediadora y los otros participantes.
} 
cuestión ambiental? y ¿cuáles son las causas y consecuencias del CC? Los grupos han tenido treinta minutos para lectura de los textos y discusión; a partir de esto, los participantes fueron invitados por la mediadora a presentar lo que cada grupo discutió. Esta etapa de la actividad tuvo duración de 48 minutos.

El contexto extraverbal común de los actores partícipes, durante el tiempo de la dinámica de grupo en la cuestión ambiental fue el auditorio y los alrededores de un Parque Municipal de Medio ambiente en Macaé, Rio de Janeiro, Brasil (como en el primer momento de esta actividad, los participantes podían elegir otras áreas del parque para hablar). En el auditorio, las sillas estaban dispuestas en un gran círculo. Los participantes sabían que esta interacción iba a ser observada, grabada y posteriormente analizada. Además, los participantes ya se conocían, ya que esta fue la tercera reunión del taller.

La grabación del video fue toda asistida, por consiguiente demarcamos en cual momento realizaríamos la transcripción, siendo este definido con el aparecimiento del conflicto entre los participantes, específicamente de los sujetos de la investigación (João, Ana y María). Esta etapa demarcada tuvo un total de 16:45 minutos (el cual se inició en el minuto 32:04 de la dinámica).

Luego de obtener los datos, estos fueron transcritos. No obstante, solo los enunciados de João, Ana y María fueron considerados en este trabajo para nuestro análisis. Por tanto, los criterios de selección de los enunciados de los sujetos de la investigación fueron: el surgimiento de conflictos entre los investigadores y los profesores, hasta el cierre de la actividad y la cuestión del sujeto que hablaba. Por otro lado, en el análisis de este trabajo, lo que los otros participantes han expresado en la interacción fue considerado en la descripción del momento en que los sujetos de esta investigación enunciarán para mejor comprensión y análisis de los sentidos de CC. Ya que, los enunciados son respuestas y sus significaciones solo pueden expresarse en la interacción. Además, los enunciados de João, Ana y María fueran demarcados de la siguiente forma: el inicio, cuando el hablante toma la vez e inicia la enunciación; el final, cuando termina su enunciado u otro hablante lo interrumpe, en otras palabras, es en la alternancia de enunciación de los hablantes lo que demarca cada enunciado.

Después de esta demarcación, se hizo un recorte que fue analizado siguiendo, paso a paso, el dispositivo analítico bakhtiniano: 1) Identificación de redacción; 2) lectura preliminar de la declaración, 3) Descripción del contexto extraverbal y 4) análisis de la redacción (VENEU et al., 2015).

El corpus de este trabajo consta de dieciocho enunciados. Agrupamos los dieciocho enunciados en el orden en que los actores hablaran en la interacción (por ejempló, el enunciado 1 fue el primero, el 2 el segundo y así sucesivamente). Los análisis se basaron en los significados atribuidos a los enunciados seleccionados a partir de cuestiones de CC, sus causas, consecuencias y relaciones entre diferentes áreas del conocimiento para mitigación de impactos.

\section{Los sentidos atribuidos a la cuestión ambiental en el contexto del CC}

Durante la interacción se observó que la alternancia entre los actores partícipes ocurrió de distintas formas. Observamos casos en que el hablante iniciaba el habla de manera espontánea, y finalizaba sin interrupciones y por su propia voluntad. Otro tipo de interacción, el habla era interrumpido y otro orador empezaba a hablar. También, hemos observado conversaciones que se iniciaron a través de la invitación, donde uno de los participantes o el mediador llamaba a la interacción con otro participante directamente, enunciando su nombre, o indirectamente, por una opinión contraria o favorable a lo argumentado anteriormente.

A partir del análisis de los enunciados de cada participante en la interacción fue posible categorizar dos sentidos atribuidos a la cuestión ambiental en el contexto del CC, que fueron: sentidos vinculados a los cambios en ecosistemas y a conocimientos científicos y sentidos que 
consideran aspectos políticos y culturales del CC. También, dichos sentidos nortearan la organización de nuestros resultados y están relacionados con los enunciados emitidos por cada participante.

\subsection{Sentidos de Cambio Climático vinculados a cambios en ecosistemas y a conocimientos científicos}

En el grupo 1, quien habló primero fue João, quien comenzó hablando acerca de la dificultad del grupo en entender la relación entre las preguntas y el texto 1, pues no les quedó muy claro. En efecto, João no comprendió qué aspectos de la cuestión ambiental se esperaba en la discusión como pode ser visto en el fragmento del Enunciado (E) 1:

[...] creo que, en realidad, lo que más me fijé en esa primera parte, fue la primera pregunta, que creí que la cuestión planteada no era muy clara. Cuando usted [para la mediadora] habla así: ¿Qué es la cuestión ambiental?... yo no logré a entender entendía si era una discusión respecto a la problemática ambiental o si para nosotros lo que es el ambiente. Para mí no quedó claro. (JOÃO, 2016 - E1, Líneas 1- 6, traducción del portugués).

A pesar de reflexionar esta crítica, considera que el grupo ha discutido a partir de la cuestión de las consecuencias de la problemática ambiental en los ambientes naturales. En este sentido, João describe aspectos generales del texto, enfatizando que el texto se trataba más bien de una historia sobre la percepción de las personas en las zonas rurales del Cariri cearense, en Brasil, que sufren consecuencias directas del CC. En este sentido, parece minimizar la importancia de la percepción de las personas y de la información planteada en el texto, una vez que el mismo no trata de enseñar aspectos del $\mathrm{CC}$ sus causas y consecuencias con dados científicos, con informaciones de papers.

Tanto es así, que João buscó una respuesta correcta y real en las cuestiones de la dinámica, al emitir sus enunciados, justificándose en la ausencia de fundamento de la literatura académica, porque los textos eran sólo contextos, generando la idea que los textos de la actividad no estaban elaborados a partir de conocimientos científicos producidos por una comunidad científica, tratándose de relatos personales y, por tanto, no podrían ser considerados para la reflexión en la dinámica. Así que, João estuvo buscando encontrar una respuesta a la pregunta acerca de la cuestión ambiental y del CC basada en la literatura académica.

Ana también ha presentado enunciados vinculados a los aspectos del conocimiento científico del CC. Sin embargo, la actividad fue planteada de modo a posibilitar el diálogo con diferentes abordajes sobre los impactos del CC. Tanto es así, que, además de cuestiones políticas, históricas, culturales y económicas, hemos buscado informaciones tecnicocientíficas por medio de datos presentados en las gráficas y en fragmentos de los textos. Así, a pesar del texto 1 presentar narrativas de historias personales, también muestra la voz de la ciencia a través del discurso de un científico, cuando presenta:

[...] El científico de la Universidad de Brasilia, Nathan Debortoli, enseña que la sequía es parte de la variabilidad climática natural del Semi árido. Sin embargo, con el cambio climático, es muy probable que sequia como esa sean más comunes e intensas en un futuro próximo [...] (Traducido del texto1 de la actividad, que fue adaptado de LINDOSO, 2013)

Asimismo, los investigadores no han considerado esta información como de relieve para basar sus discusiones, y solicitaron, por medio de sus enunciados, más base científica para los textos. En síntesis, estos resultados nos permiten comprender cómo la interacción entre sujetos, que pertenecen al mismo grupo social (los investigadores en ecología) y que comparten las formas de pensar construidas históricamente y socialmente, influye directamente en la enunciación y por ende en los sentidos que son elaborados. 
Efectivamente, el planteamiento de los investigadores respecto al conocimiento científico puede ser relacionado a visiones de la EA vinculadas a la producción del conocimiento científico para comprensión de la cuestión ambiental y toma de decisiones. Como ejemplo, podemos destacar la influencia del ambientalismo en asignaturas como ecología y conservación durante el proceso de consolidación del campo de la EA en la década de 1980 (en Brasil), generando un perfil del área de la EA, en esta década, predominantemente conservacionista, con más destaque para el ambiental (natural) (LAYRARGUES; LIMA, $2011)^{8}$.

Cuando analizamos el conjunto de los enunciados de João en la interacción, observamos que, además de la valorización de los conocimientos científicos, sus enunciados privilegian los aspectos ecológicos del CC. João relaciona los impactos del CC como quemas y liberación de gas carbónico por la agricultura, siendo estos una presión antrópica sobre los ecosistemas ejercida por los sujetos de los relatos como se puede observar no E12:

[...] sabes que no creo que él [el sujeto del texto] se ve como parte del medio ambiente es porque en ningún momento habla de las causas, que por ejemplo aquí [en el texto] la mujer trabaja con agricultura...[...] para sembrar alguna cosa hay que deforestar un área gigante, es decir, parte de la deforestación libera gas carbónico. Hay quemas etcétera. Lo que quiero decir es que ella no ve aquello, que su acción en la situación planteada, puede empeorar el problema del clima. (JOÃO, 2016 - E1, Líneas 1-5, traducción del portugués).

Ana, por su vez, también limita las causas del CC a presiones antropogénicas sobre los ecosistemas como el aumento de la liberación de $\mathrm{CO}_{2}$ por combustión de biomasa y quema de combustibles, como en el E15:

El CO2 producido por la respiración de los seres vivos, aun así no es la principal causa...lo que pasa es la liberación de biomasa acumulada, la quema de combustibles fósiles, lo que hace con que haya una acumulación de gases de efecto invernadero (ANA, 2016 - E17, Líneas 1 - 4, traducción del portugués).

Sin embargo, en los enunciados de Ana no hay solamente planteamientos relacionados a los factores antrópicos de causas del CC sobre los ecosistemas, sino que ella apunta modos de enfrentar dichas cuestiones. En sus enunciados, Ana plantea soluciones individuales, como cambios en el modo de consumo en la sociedad, que podrían ser relacionadas a visiones de EA que replantean la relación del ser humano con la naturaleza. El enunciado de Ana puede ser observado en E17:

[...] en el contexto del cambio climático, sabemos que el consumo, el estilo de vida, tener auto, eso sí, del consumo de combustibles fósiles para alimentar a la industria que...que subsidia un estilo de vida (ANA, 2016 - E15, Líneas 2 - 4, traducción del portugués).

Aunque los participantes no hayan establecido relaciones directas con la EA, entendemos que con la comprensión de la complejidad ambiental, los aspectos ecológicos y del conocimiento científico podrían ser parte de una estrategia de enfrentamiento de los impactos negativos del CC en procesos educativos vinculados a la cuestión ambiental. En realidad, algunos autores como Guimarães y Vasconcelos (2006), Carvalho (2007), Sauvé (2010) y Wals et al (2014) proponen acercamientos entre EA y EC como posibilidad de asociar el conocimiento científico proveniente de la EC a las cuestiones transdisciplinares de la EA, que visan el replanteamiento de modelos sociales, políticos y económicos que generan problemas socioambientales.

\footnotetext{
${ }^{8}$ Actualmente, este perfil del área ha dado espacio a abordajes más sociopolíticos de la EA aunque, todavía, se encuentre la vertiente conservacionista en muchos procesos educativos.
} 


\subsection{Sentidos que consideran aspectos políticos y culturales del Cambio Climático}

María plantea aspectos generales del texto y señala que las cuestiones presentadas por el grupo 1 y 2 son muy similares con la diferencia del lugar donde se denunciaban los impactos del CC (la diferencia entre de los textos del grupo 1 sería el tema de la sequía en Brasil y en el grupo 2 el derretimiento de los glaciares en Bolivia). Cuando relata el texto, María enumera las preguntas acerca del tema ambiental y las causas y consecuencias del CC. Su énfasis es en la explotación del patrimonio natural de los territorios por parte de las empresas de otros países y en la desigualdad entre los países (Norte vs Sul). En sus enunciados, plantea que los impactos del CC están relacionados a distintas dimensiones de la cuestión ambiental, como muestra en los E2 y E14:

[...] yo vi el texto muy claro como un caso de las consecuencias se pudo observar una cuestión real de todas las consecuencias $[\mathrm{del} C \mathrm{C}]$, las causas negativas y que sí estamos involucrados y somos unos de los principales causadores [del CC] (MARÍA, 2016 - E2, Líneas 13 - 16, traducción del portugués).

[...] cuando él [João] plantea que las personas deben deforestar áreas gigantes, yo veo que el texto relata personas que no son personas que deforestan sitios gigantes. Es para su supervivencia. Así las personas que viven de supervivencia, establecen una relación más directa [con la naturaleza]. Tanto es así que en el texto el presenta: las personas lastiman la ruptura hunda Pachamama que para ellos es la madre Tierra. El considera: esta es una cultura muy relacionada a la tierra. En nuestra cultura, la persona es una persona en el contexto del rural, del sol, de la tierra y del agua. Así en texto se plantea mucho eso. A lo mejor en los otros, las personas no hayan puesto de relieve la cuestión cultural de la relación con la tierra. Sin embargo, usted [al João] dijo que si un muchacho lastima la pérdida del ganado no es un grande trabajador de la industria pecuaria, porque la cuestión central para un grande pecuarita es de lucro. Esto no significa hambre, significa desventaja, significa falta de lucro pero no un modo de acabar con forma vida de él. Creo que esta es una diferencia para observar quienes están impactando más, quienes están impactando menos, quienes hacen la gran agricultura (MARÍA, 2016 - E14, Linhas 4 - 16, traducción del portugués).

Por medio del enunciado E14, hemos analizado que María plantea cuestiones que van más allá de los aspectos naturales del $\mathrm{CC}$, discutiendo temas relacionados a las injusticias y desigualdades, así como el uso de los territorios y cuestiones culturales de los pueblos Andinos de Bolivia, como presentado en el segundo texto. Las cuestiones culturales de reconexión con la tierra, planteadas por María a partir de relaciones que su grupo estableció con el texto, permiten comprender situaciones de grupos en vulnerabilidad que ya sufren impactos negativos del CC aunque hayan desarrollado una relación de conexión con la madre tierra.

En Camargo y Tonso (2013) podemos observar el planteamiento de la Constitución Política del Estado de Bolivia, que establece como deber constitucional garantir y reconocer los valores y saberes de los pueblos andinos en sus principios, ya que incorporan la organización social de los pueblos, respetando sus costumbres culturales, además de plantear la necesidad de reafirmar y unificar las naciones. A su vez, tiene el principio Vivir Bien (Sumak Kawsay), que ha sido considerado como una alternativa a los problemas socioambientales, además de rescatar las tradiciones andinas originarias, por tanto, ha sido reconocido a nivel nacional y por los movimientos sociales indígenas de Bolivia. Aunque los participantes no hayan profundizado en dichas cuestiones, María parece razonar diferentes racionalidades para entender el CC, puesto que consideró los relatos de sujetos sobre los problemas del CC en la construcción de estos sentidos, indo más allá de una mirada restricta a la ciencia y a la búsqueda de datos científicos.

Ana considera que las informaciones presentes en las gráficas distribuidas a los grupos son más relacionadas a aspectos sociales de la cuestión ambiental de las causas del CC y al modo como los países (América del Norte) que emiten más $\mathrm{CO}_{2}$ son menos vulnerables a estos 
efectos en relación con los países que emitan menos gases de efecto invernadero. En su charla, destaca la falta de preparación de los países emergentes como una de las causas del aumento de la vulnerabilidad a los impactos del CC. Ana y María parecen estar de acuerdo sobre la percepción de los temas del texto en relación a los impactos del CC y de acuerdo que hay una relación entre aspectos socioculturales y ecológicos para la comprensión de la cuestión ambiental y para mitigación del CC.

Cabe añadir que María, debido a su propia formación como profesora de ciencias biológicas, no desconoce aspectos ecológicos como impactos de deforestación, devastación ambiental y contaminación de los ecosistemas relacionados al CC. En este sentido, añadir aspectos socioculturales a los procesos educativos permite un abordaje crítico de la EA. Así, en esta visión, la EA juega un papel importante en la comprensión de los factores y contradicciones que sustentan las injusticias, conflictos y diferentes proyectos de sostenibilidad y aporta modos de enfrentarlos y cambiarlos.

Autores como Hart (2009) defienden nuevos abordajes críticos de la EA hacia las contradicciones de las sociedades actuales, enfatizando los casos de las injusticias sociales y ambientales, los cuales se sustentan por la lógica del desarrollo capitalista, que ignora, justifica y/o legitima la exploración y la sumisión de diversos grupos sociales a riesgos, daños ambientales y degradación socioambiental. En el caso del CC, dichos planteamientos críticos pueden considerar las diferencias entre grupos de actores sociales más vulnerables al CC y grupos que se benefician con los impactos. Como por ejemplo, en los textos utilizados para discusión la carga de los daños ambientales del desarrollo se concentra, predominantemente, en las poblaciones pobres y sus localidades de vida en Brasil, Bolivia y África del Sur.

De esta forma, la relación entre EC y EA, de acuerdo a esta visión, buscaría superar dicotomías entre ser humano-naturaleza, sociedad-naturaleza, y generar una concepción de ambiente que va más allá de una mirada natural. Asimismo, comprende que muchos usos de la naturaleza, diversas veces, sirven solamente a los intereses del capital. Además, la EC en asociación con la EA podría elaborar estrategias para respetar y dar voz a las características ambientales, culturales, sociales, políticas y económicas de las personas involucradas en las cuestiones ambientales.

\section{Consideraciones finales}

Tanto la profesora como los investigadores han establecido relaciones entre la cuestión ambiental y el CC, en cuanto a sus causas y consecuencias. Sin embargo, observamos que los investigadores en ecología presentaron, en sus enunciados, aspectos de las causas y consecuencias del CC más vinculados a los aspectos ecológicos, como degradación de ecosistemas, desforestación, emisión de $\mathrm{CO}_{2}$. Por otro lado, la profesora planteó que las cuestiones culturales y políticas están interrelacionadas a las causas y consecuencias de CC. En cambio, no logró profundizar explicaciones sobre cómo y qué relaciones causales hay entre dichas cuestiones y el CC.

Con todo, los sentidos que establecemos a partir de los análisis de los enunciados son múltiples e híbridos, y el conflicto en la interacción permite que se pongan de relieve uno u otro enunciado que permite establecer sentidos de acuerdo o en contra a lo planteado por otro participante. Asimismo, el presente estudio nos permitió reflexionar respecto a la discusión del tema ambiental, generando discursos que transitan entre temas que pueden ser trabajados a partir de perspectivas de EA y científicas, como señala Tozoni-Reis (2008), revelando, así, que las cuestiones ambientales pueden ser miradas en la interfaz entre la EA y la EC. A partir de esto, cuando se trata de la cuestión ambiental en la enseñanza de las ciencias y en la formación de los profesores, el vínculo entre EA y EC puede contribuir con una formación que empodere a los profesores de ciencias a abordar las cuestiones ambientales a partir de relaciones con 
diferentes áreas del conocimiento; a partir de cuestionamientos respecto a controversias sociocientíficas y de los desafíos que implican los problemas ambientales actuales.

En este sentido, la formación docente con los aportes del campo de la EA plantea que para el enfrentamiento de la cuestión ambiental es preciso la superación de una mirada solamente restricta a la ciencia, y, por ende, necesita otros conocimientos, experiencias, visiones e ideas - con esto son necesarios el reconocimiento y la legitimación de otros saberes (no académicos), en los diferentes espacios participativos, debido a la diversidad de actores y la multidimensionalidad de los problemas ambientales contemporáneos (LIMA, 2009; SAUVE, 2010; LANG et al., 2012).

\section{Referências}

VOLOCHINOV, V. N., BAKHTIN, M. Discurso na vida e discurso na arte: (sobre poética sociológica). Trad. Carlos Alberto Faraco e Cristóvão Tezza [para fins didáticos]. Tradução baseada em Discourse in Life and Discourse in Art - Concerning Sociological Poetics. In: VOLOSHINOV, V. N. (Org.). Freudism. New York: Academic Press, 1976. p. 1-25.

BAKHTIN, M. Marxismo e filosofia da linguagem. 12 ed. São Paulo: Hucitec, 2006.

CAMARGO, T. D.; TONSO, S. Educação ambiental crítica e epistemologia do sul: reflexões sobre o "vivirbien" (suma qamaña). In: ENCONTRO DE PESQUISA EM EDUCAÇÃO AMBIENTAL, Rio Claro. Anais do VII Encontro de Pesquisa em Educação Ambiental, p.1-16, 2013.

CARVALHO, L. M. O discurso ambientalista e a educação ambiental: relações com o ensino das ciências da natureza. In: ENCONTRO NACIONAL DE PESQUISA EM EDUCAÇÃO DE CIÊNCIAS, VI ENPEC, 2007, Florianópolis. Atas... Florianópolis: Abrapec, 2007. p. 1-13. Disponível em: http://www.nutes.ufrj.br/abrapec/vienpec/search0.html.

CASAGRANDE, A.; SILVA JUNIOR, P.; MENDONÇA, F. Mudanças Climáticas e aquecimento global: controvérsias, incertezas e a divulgação científica. Revista Brasileira de Climatologia, Curitiba, v.8, n.1, Ano 7, jan./jun.. 2011.

FREIRE, L. M.; CÁCERES, M. A. M.; JULIANI, S. F. Formación de profesores de ciencias desde la perspectiva ambiental: experiencias de investigación. Madoquim: Memorias de La Maestría En Docencia de La Química, Colômbia, v. 5, n. 4, p.23-30, 2016.

GUIMARÃES, M.; VASCONCELLOS, M. das M. N. Relações entre educação ambiental e educação em ciências na complementaridade dos espaços formais e não formais de educação. Educar em Revista, Curitiba, v.1, n.27, p. 147-162, 2006.

HART, P. Em resposta a "isto não é bom o suficiente": reflexões sobre a pesquisa em educação ambiental no Canadá em contextos de expansão. Educação em Revista, Marília, v. 25, n. 3, p. 159-190, 2009.

LANG, D. J., Wiek, A., Bergmann, M., Stauffacher, M., Martens, P., Moll, P., Swilling, M., Thomas, C.J. Transdisciplinary research in sustainability science: practice, principles, and challenges. Sustainability Science, v.7, n.1, p. 25-43, fev., 2012.

LAYARARGUES, P. P.; LIMA, G. F. D. C. Mapeando as macrotendências político-pedagógicas da educação ambiental contemporânea no Brasil. Ambiente \& Sociedade, São Paulo, v.17, n.1 p. 23-40, jan./mar., 2011.

LAYRARGUES, P. P.; LIMA, G. F. D C. As macrotendências político-pedagógicas da Educação Ambiental Brasileira. Ambiente \& Sociedade, São Paulo, v. 17, n. 1, p.23-40, jan. 2014. 
LEFF, E. Complexidade, Racionalidade Ambiental e Diálogo de Saberes. Educação \& Realidade, Porto Alegre, v. 34, n.3, 2009.

LIMA, G. F. C. Mudanças climáticas e conservação social: riscos do aquecimento global. Gaia Scientia, João Pessoa, v. 3, n.1, p. 35-46, 2009.

LIMA, G. F. C. Educação Ambiental e Mudança Climática: convivendo em contextos de incerteza e complexidade. Ambiente \& Educação, São Paulo, v. 18, n. 1, p.91-112, 2013.

LINDOSO, D. P. Vulnerabilidade e adaptação da vida às secas: desafios à sustentabilidade rural familiar nos Semiáridos Nordestinos. 519 f. 2013. Tese (Doutorado em Desenvolvimento Sustentável) - Universidade de Brasília, Brasília, 2013.

OLFARNES, T.; GITOW, A. Melting glaciers alter a way of life: adapting to harsh, new realities in Bolivia. United Nations Population Fund., 2009. Disponible en: <http://www.unfpa.org/ public/media_resources/swp09>. Acceso en: março 2016.

PIRES, V. L. Dialogismo e alteridade ou a teoria da enunciação em Bakhtin. Organon, Rio Grande do Sul, v. 16, n. 32, p.1-2, jan. 2002.

SÁ, E. F. de et al. Discutindo a objetividade na pesquisa em educação em ciências. Ciênc. Educ., Bauru, v. 20, n. 2, p. 411-431, 2014.

SAUVE, L. Educación científica y educación ambiental: un cruce fecundo. Enseñanza de las ciencias, v.28 n.1, p. 5-18, 2010.

SOUZA, S. J.; ALBUQUERQUE, E. D. P. A pesquisa em ciências humanas: uma leitura bakhtiniana. Bakhtiniana, v. 7, n.2, p. 109-122, jul/dez, 2012.

TOZONI-REIS, M. F. C. Formação dos educadores ambientais e paradigmas em transição. Ciência \& Educação, Bauru, v. 8, n.1, p.83-96, 2002. Disponível em: <http://www.scielo.br/pdf/ciedu/v8n1/07.pdf >. Acceso en: março de 2016.

TOZONI-REIS, M. F. C. Educação Ambiental: natureza, razão e história. 2 ed. Campinas: Autores Associados, 2008.

UNESCO. Mudança climática em sala de aula: curso da UNESCO para professores secundários (fundamental II e ensino médio) sobre educação em mudança climática e desenvolvimento sustentável (EMCDS) / David Selby e Fumiyo Kagawa. Brasília: UNESCO, 2014. Disponível em: <http://unesdoc.unesco.org/images/0022/002297/229737por.pdf〉. Acceso en: março de 2016.

VENEU, A. et al. Análise de discurso no ensino de ciências: considerações teóricas, implicações epistemológicas e metodológicas. Revista Ensaio, Belo Horizonte, v. 1, n. 17, p.126-149, Jan. 2015.

VENTURA, G.; FREIRE, L. M. . A Educação em Ciências e as mudanças no mundo produtivo no cenário contemporâneo: contribuições para o enfrentamento da questão ambiental. In: ENCONTRO NACIONAL DE PESQUISA EM EDUCAÇÃO EM CIÊNCIAS, 10, 2015. Águas de Lindóia. Anais... Águas de Lindóia: ABRAPEC, 2015. Disponível em http://www.abrapecnet.org.br/enpec/xenpec/anais2015/resumos/R1118-1.PDF. Acesso en: março de 2016.

WALS, A. E. J. et al. Convergence between Science and Environmental Education. Science Education, Washington, v.344, n.1, p. 583-584, mai. 2014. 